\title{
A new hybrid GA-PSO method for solving multi-period inventory routing problem with considering financial decisions
}

\author{
Masoud Rabbani, Milad Baghersad, Ruholla Jafari \\ College of engineering, University of Tehran (Iran) \\ mrabani@ut.ac.ir,milad_baghersad@ut.ac.ir,rubolla.jafari@ut.ac.ir
}

Received: November 2012

Accepted: August 2013

\section{Abstract:}

Purpose Integration of various logistical components in supply chain, such as transportation, inventory control and facility location are becoming common practice to avoid suboptimization in nowadays' competitive environment. The integration of transportation and inventory decisions is known as inventory routing problem (IRP) in the literature. The problem aims to determine the delivery quantity for each customer and the network routes to be used in each period, so that the total inventory and transportation costs are to be minimized. On the contrary of conventional IRP that each retailer can only provide its demand from the supplier, in this paper, a new multi-period, multi-item IRP model with considering lateral trans-shipment and financial decisions is proposed as a business model in a distinct organization. The main concern of this paper is to propose a new decision making approach usable for an organization to decide economically whether establish a new agent.

Design/methoddogy/approadr The problem is formulated as a Mixed Integer Linear Programming (MILP) model. A new hybrid genetic algorithm (GA)-particle swarm optimization (PSO) metaheuristic algorithm is proposed which showed to be applicable and reliable comparing its numerical results with GAMS. Finally, a decision procedure with three phases is proposed to help an organization to find whether establishing a new agent has economic justification or not. 
Findings: Numerical results of new proposed algorithm comparing with GAMS are showed that the proposed algorithm produce good answers and the unique chromosome represented for the proposed solving methodology is adaptive with the essence of IRP.

Originality/value Motivated by real world and analyzing gap in literature, a new MILP model for multi-period, multi-item IRP with considering lateral trans-shipment and financial decisions is proposed and then the model is solved with a new hybrid GA-PSO meta-heuristic algorithm. The chromosome represented for the proposed solving methodology is unique and is another contribution of this paper which showed to be adaptive with the essence of IRP problem.

Keywords: inventory routing problem, lateral trans-shipment, genetic algorithm, particle swarm optimization, hybrid meta-heuristic

\section{Introduction}

Nowadays in manufacturing business, especially supply chain issues, there is a rising need for efficient behavior because of competition and reduced profit margins. Also production actors and service providers in the transport industry are facing a more challenging situation compared to last decades. In fact, they have to plan the benefit of the whole chain instead of their own company. Since transportation costs are one of the main supply chain costs, vehicle routing problem (VRP) attracted much interest. However inventory and transportation costs are in conflict. The integration of transportation and inventory decisions in literature is represented by IRP. It is to determine the delivery quantity for each customer and a set of feasible vehicle routes for the delivery of the quantities in each period, subject to the vehicle capacity constraints and the customers' product requirements and inventory capacity constraints, so that a total inventory and transportation cost is minimized.

The main concern of this research is to propose a practical IRP model considering simultaneously lateral trans-shipment and financial decisions in order to help an organization to decide economically whether establish a new agent. Consequently, a novel MILP formulation is proposed to model the problem. To solve the proposed model, for the first time, a new hybrid GA-PSO meta-heuristic algorithm is developed and some randomly generated test problem is provided to demonstrate the usefulness of the proposed hybrid algorithm. A comparison between hybrid algorithm results and GAMS solutions indicates that the average gap is low and the proposed hybrid method gives totally reliable and good answers.

The rest of the paper is structured as follows. Section 2 provides a review of the related literature whereas Section 3 presents the related soft computing techniques. Mathematical formulation, definition and assumptions of the concerned IRP problem are put forward in 
Section 4. Also the specific coding that is used for solving this problem is described in Section 5. Section 6 presents the proposed methodology output and financial analysis, and finally, the discussing and conclusion are provided in Section 7.

\section{Literature Review}

As pointed by Andersson, Hoff, Christiansen, Hasle and Løkketangen, (2010), approaches for dealing with IRP vary depending on features such as time, demand, topology, vehicle fleet, inventory, and solution method. In an interesting survey, Moin and Salhi (2007) gave a logistical overview of IRPs. They classified the literature according to the number of time periods, single-period, multi-period and infinite horizon models. In another paper, Andersson et al. (2010) described industrial aspects of combined inventory management and routing in maritime and road-based transportation, and gave a classification and comprehensive literature review of this research area. Here we just review the related IRP papers, for further information the reader can refer to the mentioned review papers.

Because of the complexity of IRPs, only small instances of IRPs can be solved optimally. Therefore, almost all solution approaches proposed in the literature are heuristics, either pure heuristics or optimization methods ended before proven optimality (Andersson et al., 2010). The most heuristics proposed for the multi-period inventory routing problem, among others (Campbell \& Savelsbergh, 2004), are based on decomposing the problem into hierarchical sub-problems. They saw IRP as one allocation problem, where the decisions are how much and when to deliver to the customers, and one routing problem where the routes are determined. Abdelmaguid, Dessouky and Ordóñez (2009) addressed the inventory routing problem with backlogging in which multi-period vehicle routing and inventory holding and backlogging decisions for a set of customers are to be made. They developed constructive and improvement heuristics to obtain an approximate solution for the problem and demonstrated their effectiveness through computational experiments. Bard \& Nananukul. (2010) developed a mixed-integer programming (MIP) model aimed at minimizing production, inventory, and delivery costs. They assumed single production facility, a set of customers with time varying demand, and a fleet of homogeneous vehicles. They solved the underlying MIP with a hybrid methodology that combines exact and heuristic procedures within a branch-and-price framework, and showed that the hybrid scheme can solve instances with up to 50 customers and 8 time periods within 1 hour. Bertazzi, Bosco, Guerriero and Laganà (2011) studied an inventory routing problem in which a supplier has to serve a set of retailers and for each retailer, a maximum inventory level is defined and a stochastic demand has to be satisfied over a given time horizon. They proposed a hybrid rollout algorithm for the solution of the problem and its performance is evaluated on a large set of randomly generated problem instances. 
In conventional IRP, each retailer can only provide its demand from supplier but in the model presented in this paper, retailers also can have lateral trans-shipment. In other words, each retailer can provide its demand from other retailers. Paterson, Kiesmuller, Teunter and Glazebrook (2011) presented a review in inventory models with lateral trans-shipments. This review has shown that lateral trans-shipments have been applied in many different types of inventory system in a varied range of industries. Examples of such models in the literature are Fredrik (2010) and Fredrik (2009). Shen, Chu, and Chen (2011) solved a maritime IRP with trans-shipment in crude oil transportation. They proposed that crude oil can be transported from the depot, either directly or via trans-shipment ports at the input and output ports of a pipeline to costumer harbors to satisfy dynamic demands of the customers. Coelho, Cordeau and Laporte (2012) presented an IRP that allows trans-shipments, either from the supplier to customers or between customers. They considered a multi period IRP with a supplier and a set of customer, where both the supplier and customers incur inventory holding costs. Demand of customers is deterministic and inventories are not allowed to be negative. They assume that only a single vehicle is available. They proposed an adaptive large neighborhood search heuristic to solve the problem. This heuristic manipulates vehicle routes while the remaining problem of determining delivery quantities and trans-shipment moves is solved through a network flow algorithm. Their approach can solve four different variants of the problem: the IRP and the IRP with trans-shipment, under maximum level and order-up-to level policies.

There is a massive gap in the literature on IRPs with considering financial decisions. In a related paper, Chen \& Lin. (2009) addressed a multi-period supply chain network design problem with considering several aspects of practical relevance such as those related with the financial decisions that must be accounted for by a company managing a supply chain. They considered decisions to be made comprise the location of the facilities, the flow of commodities and the investments to make in alternative activities to those directly related with the supply chain design. They formulated the problem as a multi-stage stochastic mixed-integer linear programming problem, aimed with maximizing the total financial benefit. They also discussed about a methodology for measuring the value of the stochastic solution in the problem.

In this paper, a new decision making approach for an organization, which its main aim is to produce some items in a firm and to sell them through its agents, is proposed to decide economically whether establish a new agent. Reviewing the literature we contemplate there has been never such an approach in IRP deciding to establish a new retailer (agent) or not with considering possibility of lateral trans-shipment. Consequently, a new MILP model for multi-period, multi-item IRP with considering lateral trans-shipment and financial decisions is proposed. Although different solution methods are proposed in the literature to solve IRPs, considering lateral trans-shipment and financial decisions in the proposed model-which adds 
up to the complexity of proposed IRP model-spells a need for a new solution method. Therefore, a novel hybrid GA-PSO meta-heuristic algorithm is introduced to solve the proposed MILP model.

\section{Soft computing techniques}

There are a lot of well-known meta-heuristics applied to solve NP-hard problems. Furthermore, the use of these meta-heuristics (e.g., GA, SA, TS, SS and ATO) is growing more and more. Nowadays, researchers have realized that they are not just interested in solving their own-defined problem by simply using a classic meta-heuristic algorithm, but also improving and adapting that algorithm to fulfill their need to find better solution. As matter of fact this is the attractiveness of meta-heuristic algorithms; on the contrary of heuristics, they can be altered to adapt the problem which they are dealing with. It is exceptionally interesting that researchers are not just trying to alter or to justify existing meta-heuristics to have better answers in minimum consumed time. However, recently they have been exploiting more than one heuristic or meta-heuristic algorithm to acquire a more effective solving approach.

This is precisely the reason behind our applying two of the most renowned meta-heuristics, namely Genetic Algorithm (GA), and Particle Swarm Optimization (PSO). The complexity and uniqueness of the proposed problem in this paper made us study different algorithms and put them into perspective with our problem specialties just to find out their amalgamation could work well.

\subsection{Genetic Algorithm}

GA is a progressive and highly adaptive meta-heuristic algorithm which was invented by Holland and his colleagues in the 1965. Taking a glance of these years' efforts we can see how widely GA has been in use in various majors and studies. During these few decades researchers has proven to be highly absorbed into using the methodology. Just like any other recognized and highly in used algorithm it has gone through modification and alteration. We can introduce GA essence by the chromosome concept, its initialization, its crossover and mutation operators. GA is a highly adaptive algorithm; one is able to inspire the spirit of their considered problem into the procedure of the algorithm by simply defining its chromosome and fitness function. In another words, GAs are probabilistic search techniques that are inspired by Darwin's theory about evolution. In GAs, while a population of candidate solutions evolves through generations by the use of genetic operations, some individuals adapt better to the environment and have more possibilities of survival. The general structure of the genetic algorithms is described in Figure 1. 


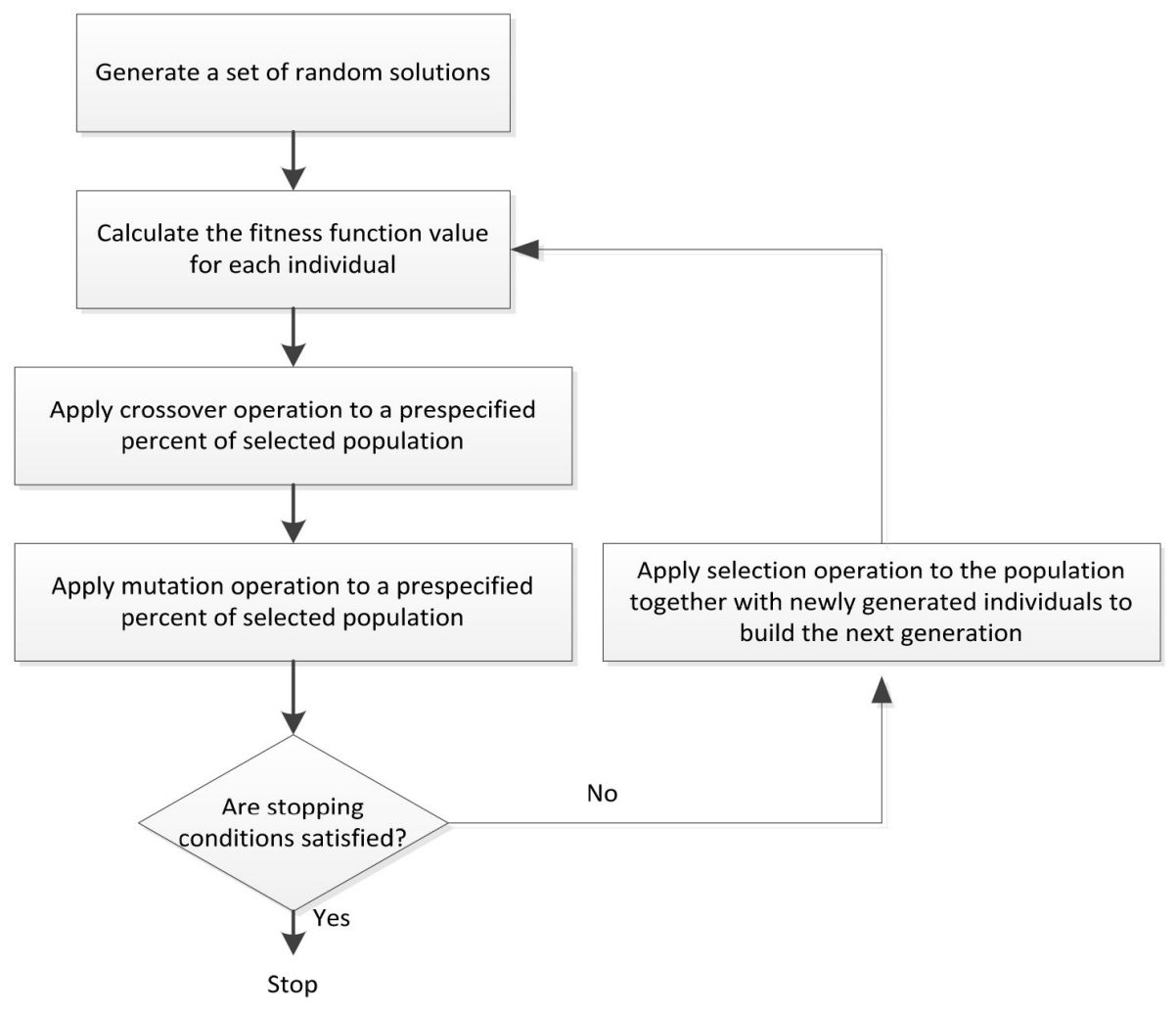

Figure 1. Flowchart of genetic algorithm

\subsection{Particle swarm optimization Algorithm}

PSO is a progressive and also evolutionary calculation algorithm introduced by (Kennedy \& Eberhart, 1995). This algorithm has been widely used over these few decades. Though their first algorithm has gone through tangible alteration and modification by other researcher or its innovators themselves, its nature has remained steady and it has been outstandingly in use along the other meta-heuristic algorithms. The algorithm has some similarity with other evolutionary algorithms. It is among population-based search algorithm and also its initialization is completely randomized. Its matchless difference is, however, each individual, called particle, efficiently keeps and uses its own-achieved best answer (pbest) during the competition.

The outline of PSO process is as follows:

1. Initialize a population of particles with uniformly distributed random positions and velocities on d dimensions in the problem space.

2. For each particle, compute the selected optimization fitness function.

3. Compare particle's fitness computation with particle's pbest. If current value is better than pbest, then set the particle's pbest value and position equal to the current.

4. Compare fitness computation with the population's overall previous best, called gbest. If current value is better than gbest, then reset gbest to the current. 
5. Change the velocity and Values of the particle according to equations (1) and (2), respectively:

$$
\begin{gathered}
v_{i d t}=C_{0} \times v_{i d(t-1)}+C_{1} \times r \times\left(P b_{i d}-X_{i d}\right)+C_{2} \times r \times\left(G b_{d}-X_{i d}\right) \\
X_{i d t}=X_{i d(t-1)}+v_{i d t}
\end{gathered}
$$

Where $\mathrm{X}$ : the position of population; $\mathrm{i}$ : index for each particle; $d$ : index for each dimension; $t$ : index for each iteration; $v$ : velocity; $r$ : a randomized number with uniform distribution $[\mathrm{U}(0,1)]$; $\mathrm{Pb}$ : the position of pbest; $\mathrm{Gb}$ : the position of gbest and C0, C1, C2 are Inertia, personal learning and global learning Coefficients, constants to be specified by practitioner.

6. Loop to step (2) until a stop criterion is met, empirically an adequately good fitness or a maximum number of iterations.

The constants which have to be specified by practitioner lead the demeanor and efficacy of the PSO Algorithm. One may deduce the role of constant, CO called Inertia Coefficient, as a desire of each particle not to change its roaming abruptly. Constants $\mathrm{C} 1$ and $\mathrm{C} 2$, called respectively personal and Global learning Coefficients, represent weighting of the stochastic motivation terms that draw each particle toward pbest and gbest values respectively. Elaboration of these two constants modifies the amount of tension in the system. Low values allow particles to rove from target area before being tugged back, since high values end in sudden movement toward objective area. Appropriate selection of the inertia coefficient cause a harmony between global and local exploration and exploitation, and results in less iterations to find a sufficient solution (Eberhart \& Shi, 2001).

\section{Problem definition and MILP model}

\subsection{Problem description and assumptions}

We consider a firm which produces several products and has a set of agents distributed around a particular city. It is been assumed that agents face with different demand for every product (item) in each time period (Dynamic Demand). Agents can store inventory for the next period regarding a holding cost, however, they have limited capacity. In this paper, shortage is permitted with penalty cost and it is treated as lost demand.

Agents mainly provide their demand from firm; however, they may provide their demand from other agents with lateral trans-shipment in case it is proven to be economical. There are two types of homogeneous vehicles with limited capacity. The first types of vehicles are used to transport products from firm to agents in the beginning of each period, and it must return to the firm at the end of the period. Also no further delivery assignments should be 
made in the same period. The second types of vehicles are employed between agents when lateral trans-shipment is required. Figure 2 illustrates an example of the proposed model.

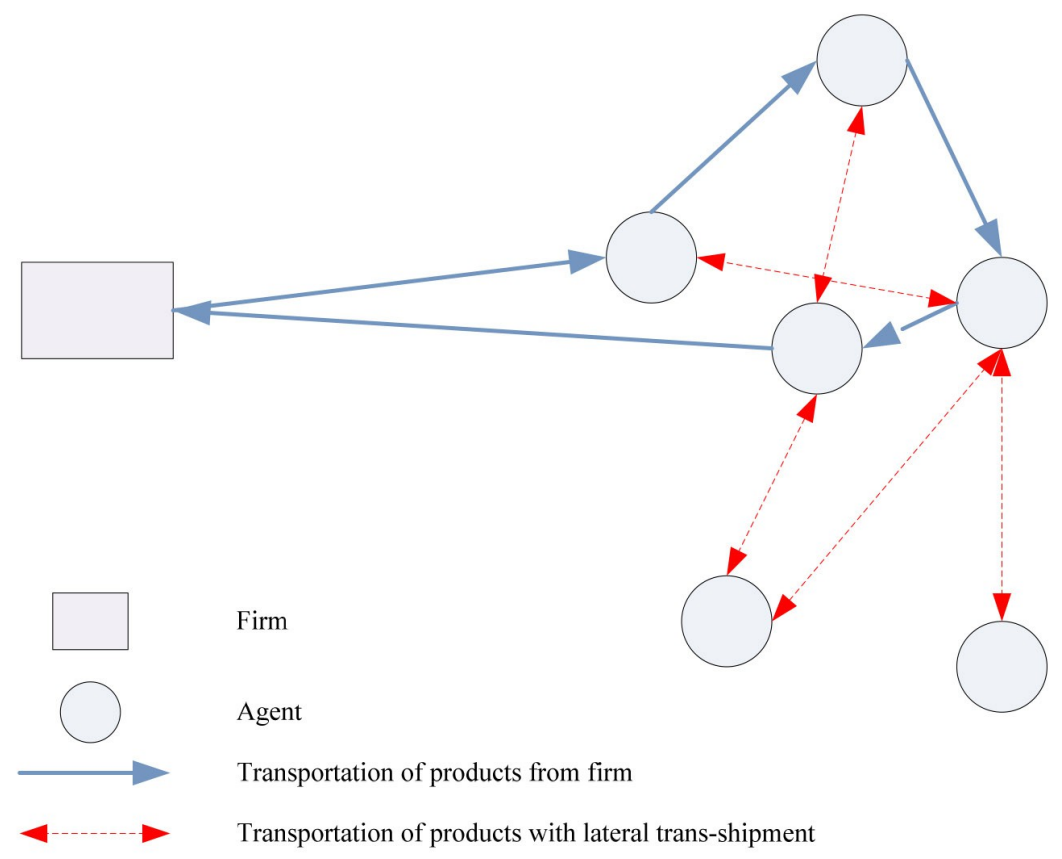

Figure 2. An example of the IRP with lateral trans-shipment

As it was mentioned, considered firm produces several items that each of them has different cost of production. Reasonably, selling of each product gains the organization a distinct profit. To formulate the model, due to the complexity of considering production cost, this model is just concerned with the gain due to selling products. This gain is actually the deduction between a product purchase price and production costs (i.e. the costs of raw materials and labor). To clarify, there are two types of costs considered in the model: the costs of inventory and transportation by which our model tries to optimize, and the production costs which are used to calculate the gain of each product.

Apart from the ability of our model to optimize the inventory and transportation costs of an organization in current situation, the model can be used in the decision making process of establishing a new agent. Suppose an organization, already optimized based on this paper's model, has decided to initiate a development project that is establishing a new agent. The demand of this new agent has been foreseen and it is dynamic, the same as the other agents. This definitely inflicts some charges on organization. These charges may be enumerated by costs related to new building, new staff, adding new production line and so on. However, required fund this new establishment may be acquired by selling bound or distributing new stock.

The fact that we engage the net profit of selling in the model, beside the transportation and inventory costs, made our model applicable to address the decision making problem explained in preceding paragraph. If we assume that the new agent is already established, the model can 
provide us the information about the exact future income considering the optimized future network. In another words, our model can be used as a mean that provides dependably accurate information about the profitability of establishing new agent. With this valuable information in hand, and considering the costs of establishing a new agent and also the different ways of funding, the organization will be able to decide effectively whether to establish a new agent or not. However, in section 6, a decision procedure with three phases is proposed to help an organization to find whether establishing a new agent has economic justification or not.

\subsection{The proposed MILP model}

\section{Notations}

The following notation is used to formulate the proposed model.

\section{Indices}

$t \quad$ Period index $(t=1, \ldots, T)$

$i, j \quad$ Agent (retailer) or firm (supplier) index, where $i, j=1, \ldots, N-1$ represent retailer, $N$ represent new agent and 0 represents the supplier

$s \quad$ Product type $(s=1, \ldots, S)$

$k \quad$ Number of first type's vehicles $(k=1, \ldots, K)$

\section{Parameters}

$P_{s} \quad$ Revenue (selling price minus purchasing cost and production cost) from the sale a unit of product $s$

$Q_{l} \quad$ Each first type's vehicles capacity

$Q_{2} \quad$ Each second type's vehicles capacity

$c_{i j} \quad$ Shipping cost along arc $(i, j)$ where $c_{i j}=c_{j i}$ and the triangle inequality, $c_{i l}+c_{l j} \leq c_{i j}$, holds for any $i, j, l$ with $l \neq i, l \neq j$

$p_{i j} \quad$ Lateral trans-shipment cost along arc $(i, j)$

$h_{i} \quad$ Holding cost per unit of product at retailer $i$ and per period

$V_{i} \quad$ Inventory capacity of retailer $i$ 
$d_{i s t} \quad$ Demand of product $s$ at retailer $i$ in period $t$

$\pi_{i s} \quad$ Shortage penalty per unit of product at retailer $i$ and per period

$i^{\prime} \quad$ Internal rate of return

$f_{t} \quad$ Inflation rate in period $t$

\section{Decision variables}

$X_{i j k t}= \begin{cases}1 & \text { If vehicle } k \text { (first type) travels directly from } i \text { to } j \text { in period } t \\ 0 & \text { Otherwise }\end{cases}$

$I_{i s t} \quad$ Inventory level of product $s$ at retailer $i$ at the end of period $t$

$B_{i s t} \quad$ Shortage quantity of product $s$ at period $t$ for retailer $i$

$q_{\text {iskt }} \quad$ Delivery quantity of product $s$ to retailer $i$ with vehicle $k$ in period $t$

$Y_{i j t}= \begin{cases}1 & \text { If we have lateral trans-shipment from } i \text { to } j \text { in period } t \\ 0 & \text { Otherwise }\end{cases}$

$T_{i j s t} \quad$ Delivery quantity of product $s$ from retailer $i$ to $j$ in period $t$ with second types of vehicles (i.e. lateral trans-shipment)

\section{Formulation}

$$
\begin{aligned}
& \operatorname{Max} Z= \\
& \sum_{t=1}^{T}\left[\frac{\left(\sum_{s=1}^{S} \sum_{i=1}^{N} P_{s}\left(d_{i s t}-B_{i s t}\right)-\sum_{s=1}^{S} \sum_{i=1}^{N} \pi_{i s} B_{i s t}-\sum_{i=1}^{N} h_{i}\left(\sum_{s=1}^{S} I_{i s t}\right)-\sum_{k=1}^{K} \sum_{j=0, j \neq i}^{N} \sum_{i=0}^{N} c_{i j} X_{i j k t}-\sum_{j=1, j \neq 1}^{N} \sum_{i=1}^{N} p_{i j} Y_{i j t}\right)\left(1+f_{t}\right)}{(1+i)^{t}}\right]
\end{aligned}
$$




\section{Subject to:}

$$
\begin{aligned}
& \sum_{k=1}^{K} \sum_{j=1}^{N} X_{0 j k t} \leq k \\
& t=1, \ldots, T \\
& \sum_{j=1, j \neq i}^{N} X_{i j k t}=\sum_{j=1, j \neq i}^{N} X_{j i k t} \\
& i=0, \ldots, N, k=1, \ldots, K, t=1, \ldots, T \\
& \sum_{j=1}^{N} X_{0 j k t} \leq 1 \\
& k=1, \ldots, K, t=1, \ldots, T \\
& \sum_{k=1}^{K} \sum_{j=0, j \neq i}^{N} X_{i j k t} \leq 1 \\
& i=1, \ldots, N, t=1, \ldots, T \\
& \sum_{k=1}^{K} \sum_{i=0, i \neq j}^{N} X_{i j k t} \leq 1 \\
& j=1, \ldots, N, t=1, \ldots, T \\
& I_{i s t-1}+\sum_{k=1}^{K} q_{i s k t}+\sum_{j=1, j \neq i}^{N} T_{j s t}+B_{i t}=I_{i s t}+\sum_{j=1, j \neq i}^{N} T_{i j s t}+d_{i s t}+B_{i s t-1} \quad j=1, \ldots, N, S=1, \ldots, S, t=1, \ldots, T \\
& \sum_{s=1}^{S} \sum_{i=1}^{N} q_{i s k t} \leq Q_{1} \\
& t=1, \ldots, T, k=1, \ldots, K \\
& \sum_{s=1}^{S} T_{i j s t} \leq Q_{2} \\
& i, j=1, \ldots, N, j \neq i, t=1, \ldots, T \\
& \sum_{s=1}^{S}\left(I_{i s t-1}+\sum_{k=1}^{K} q_{i s k t}+\sum_{j=1, j \neq i}^{N} T_{j i s t}-\sum_{j=1, j \neq i}^{N} T_{i j s t}\right) \leq V_{i} \\
& i=1, \ldots, N, t=1, \ldots, T \\
& Y_{0 j t}=0 \\
& j=1, \ldots, N, t=1, \ldots, T \\
& Y_{i 0 t}=0 \\
& i=1, \ldots, N, t=1, \ldots, T \\
& U_{i t}-U_{j t}+(N+1) X_{i j k t} \leq N \\
& i, j=1, \ldots, N, j \neq i, k=1, \ldots, K t=1, \ldots, T \\
& T_{i j s t} \leq M \cdot Y_{i j t} \\
& i, j=0, \ldots, N, j \neq i, s=1, \ldots, S, t=1, \ldots, T \\
& q_{i s k t} \leq M \cdot\left(\sum_{k=1}^{K} \sum_{j=0, j \neq i}^{N} X_{j i k t}\right) \\
& i=1, \ldots, N, s=1, \ldots, S, k=1, \ldots, K, t=1, \ldots, T \\
& I_{i s t} \leq M \cdot F_{i s t} \\
& i=1, \ldots, N, s=1, \ldots, S, t=1, \ldots, T \\
& B_{i s t} \leq M \cdot\left(1-F_{i s t}\right) \\
& i=1, \ldots, N, s=1, \ldots, S, t=1, \ldots, T \\
& X_{i j k t} \in\{0,1\} \\
& i, j=1, \ldots, N, j \neq i, k=1, \ldots, K, t=1, \ldots, T
\end{aligned}
$$




$$
\begin{array}{ccc}
I_{i s t} & 0 & i=1, \ldots, N, s=1, \ldots, S, t=1, \ldots, T \\
B_{i s t} & 0 & i=1, \ldots, N, s=1, \ldots, S, t=1, \ldots, T \\
q_{i s k t} & 0 & i=1, \ldots, N, s=1, \ldots, S, k=1, \ldots, K, t=1, \ldots, T \\
T_{i j s t} & 0 & i, j=1, \ldots, N, i \neq j, s=1, \ldots, S, t=1, \ldots, T \\
U_{i t}=1,2, \ldots, N & i=1, \ldots, N, t=1, \ldots, T
\end{array}
$$

Objective Function (3) calculates difference between revenue and costs of, respectively, shortage, inventory and transportation. Constraint (4) ensures that the number of the first type of vehicles used for delivery in each period does not exceed the total number of first type of vehicles. Constraint (5) ensures that the number of the first type of vehicles leaving from a retailer or the supplier is equal to the number of its arrival. Constraint (6) ensures that each first type of vehicles can travel maximum once in each period. Constraints (7) and (8) ensure that each retailer must be visited maximum once and with one of the first type of vehicles in each period. Constraint (9) is the inventory balance constraints of each retailer at each period. Constraints (10) and (11) ensure that loading of each first and second type of vehicles in each period does not exceed respective capacities. Constraint (12) limits the inventory level of the retailers to the corresponding storage capacity. Constraints (13) and (14) ensure that the second type of vehicles just travel among retailers. Constraint (15) eliminates sub-tours for each first type of vehicles at each period. Constraints (16) and (17) are logical relationships between $Y_{i j t}, T_{i j t}, X_{i j k t}$ and $q_{i t}$. Constraints (18) and (19) ensure that at end of each period, each retailer can't have both of inventory and shortage. Finally, constraints (20) to (25) show the type of variables.

\section{Specific Coding}

\subsection{General outline}

Contemplating previous studies, see for instance (Liu \& Chen, 2011), IRP has shown to be an intricate and NP-hard problem. Due to the mentioned complexity of IRP problem, in this paper, we assumed IRP to be composed of two sub-problems, i.e. VRP and Inventory Problem (IP). Since VRP itself is a NP-hard problem (Lenstra \& Kan, 1981; Prins, 2004), a Genetic approach, due to its proven adaptively, has been proposed to deal with indicated VRP complexity. The duty of Proposed GA is to construct vehicle routs at each period, i.e. to find an eligible solution for the first decomposed sub-problem. Another reason to employ GA approach for VRP is its powerful chromosome structure. Defining an appropriate chromosome will endow us the ability to bring VRP constraints into its structure. After routes are constructed by GA chromosome it needs to be decided how much product each agent received at each period. Since making this last decision is also intricate because of considered assumptions (such as multi periodic, multi 
items, and allowing shortage) we decided to apply PSO Algorithm to solve second subproblem. However, using a meta-heuristic algorithm nested in another one may cause a long time for solving a hybrid approach. To deal with mentioned concern, proposed PSO parameters are set to end in a rather swift solver.

Although there has been a load of efforts and research for the sake of promoting GA operators, the role of defining eligible and qualified chromosome has an impacting influence on the performance of GA. GA chromosome has the most adaptable essence among the other GA concepts, moreover, it can be claimed that it is the superiority point of GA in comparison with the other approaches. Furthermore there had been unprecedented number of unique chromosome in vast gamut of majors and researches. As for this paper, chromosome encoding has given an adaptive characteristic to the algorithm. This fact is actually the reason behind applying the algorithm to the first part of our problem. With this advantage, we managed to inspire the essence of transportation part of the problem to its chromosome. Consequently, this brought about the presentation of crossover and mutation operators.

As for the PSO, Two of the most renowned positive features of PSO are its swiftness and its usability for continuous problems. Latter addresses the continuous feature of inventory problem whereas former satisfies the need for a speedy algorithm. However, to make having another algorithm nested in the other happen we justified the PSO demeanor variables in a way that would end in an as-fast-as-possible algorithm.

\subsection{Chromosome Representation}

A matrix with $N \times 3 T$ elements is used for chromosome structure, where $N$ is the number of total agents whereas $T$ is the number of periods. Each three columns specifies each period. The first column in each period is used to distinguish which vehicle should serve which agent. Note that zero in the first column shows that the agent will not be served in the period with vehicles coming from the firm (first type vehicles). Second column is for extracting the sequence of agents in each route that the assigned vehicles will serve them, whereas third column in each period is the mean by which one can determine about lateral trans-shipments. To illustrate, an example of the chromosome representation for a problem with 5 agents and 3 periods is illustrated in Figure 3. From first column of Figure 3, we can understand that vehicle 2 should serve agents 1 and 5, and vehicle 1 should serve agents 3 and 4 in the first period. From column 2 in the first period, we see that vehicle 2 (1) should first visit agent 5 and then 1 ( 4 and then 3 ) and from third column in Figure 3 we find that a lateral trans-shipment exists from agent 2 to 3 in period 1 . The same inference may be conducted to find out the other machine sequences. However, Table 1 shows the sequence of each machine in each period for chromosome represented in Figure 3. 


\begin{tabular}{|c|c|c|c|c|c|c|c|c|c|}
\hline \multirow{3}{*}{$\begin{array}{c}\text { Agent } \\
1\end{array}$} & \multicolumn{9}{|c|}{ Period } \\
\hline & \multicolumn{3}{|c|}{1} & \multicolumn{3}{|c|}{2} & \multicolumn{3}{|c|}{3} \\
\hline & 2 & 4 & 0 & 0 & 2 & 0 & 1 & 5 & 2 \\
\hline 2 & 0 & 2 & 3 & 1 & 5 & 0 & 2 & 2 & 0 \\
\hline 3 & 1 & 5 & 0 & 1 & 3 & 1 & 0 & 3 & 3 \\
\hline 4 & 1 & 1 & 0 & 2 & 4 & 0 & 2 & 1 & 0 \\
\hline 5 & 2 & 3 & 0 & 1 & 1 & 3 & 2 & 4 & 3 \\
\hline & $\begin{array}{l}\checkmark \\
\frac{d}{\alpha} \\
\frac{\partial}{\sigma} \\
\frac{\sigma}{d}\end{array}$ & 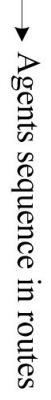 & 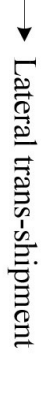 & & & & & & \\
\hline
\end{tabular}

Figure 3. Chromosome representation

\begin{tabular}{|c|c|c|}
\hline Period & Vehicle & Sequence of Agents \\
\hline 1 & 1 & Center $\rightarrow 4 \rightarrow 3 \rightarrow$ Center \\
\hline 1 & 2 & Center $\rightarrow 5 \rightarrow 1 \rightarrow$ Center \\
\hline 2 & 1 & Center $\rightarrow 5 \rightarrow 3 \rightarrow 2 \rightarrow$ Center \\
\hline 2 & 2 & Center $\rightarrow 4 \rightarrow$ Center \\
\hline 3 & 1 & Center $\rightarrow 1 \rightarrow$ Center \\
\hline 3 & 2 & Center $\rightarrow 4 \rightarrow 2 \rightarrow 5 \rightarrow$ Center \\
\hline
\end{tabular}

Table 1. Sequence of each vehicle in each period for chromosome represented in Figure 3

As it is mentioned before, this paper chromosome is unique and has never been in the literature before. However, its greatest improvement is that we have embodied the Lateral trans-shipment concept in the chromosome structure. Furthermore, the chromosome has this advantage over the previous ones that can omit an agent from receiving product from firm in a period. To put it another way, this chromosome has the ability of other renowned VRP chromosome in the literature beside the two mentioned improvements.

\subsection{GA Operators}

As mentioned earlier, one of the contributions of this paper is its new proposed chromosome, which has never been in the literature. Binary chromosomes can be simply treated by its various proposed crossover or mutation; however, a new or unique proposed chromosome forces GA's operators to be new or unique as well. In addition, in this experiment, we design the corresponding crossover and mutation Operators. The following sections are devoted to introduce them. 


\subsubsection{Crossover}

Dealing expertly with the GA, one will comprehend the essential role of the crossover operator, which is somehow the means of the GA for the communication between chromosomes. Certainly, it is the agent to converge chromosomes into the optimization. Therefore, the efficacy of the GA is highly depended on a well-operating crossover. The crossover proposed in this paper has proven to be versatile, flexible and adaptive. It uses and merges both undergoing-crossover-chromosome's (both parent) spirits to make new childes. According to the chromosome in this paper, we introduce multiple crossovers. These distinct crossovers are randomly chosen to be employed in solving procedure.

Regardless of the number agents and machines, we can introduce the proposed chromosome as a single line chromosome with tree different kind of cells (Figure 4) that are Machine Cells (first columns in each period), agent sequence cells (second columns in each period), and lateral trans-shipment cells (third columns in each period). From now on, we know this three kind of cells with respectively first, second, and third cell. The best way to introduce the applied cross over is to say we have simply used the traditional single-break crossover with minor alteration to fulfill the chromosome peculiarity. Imagine the chromosome as a single line with number of period cells, and also think of two child out of mentioned single-break crossover. That is, one of our 7 applied crossovers is just been introduced. The others are just the same with a minor difference. In introduced crossover all of the three cells (columns) were to be transformed between parents. But for other chromosome there is no need for all three cells to be transformed. [1th], [2th], [3th], [1th, 2th], [1th, 3th], [2th, 3th], and [1th, 2th, 3th] are all of the possible ways of engaging each types of cell that makes the mentioned 7 crossovers.

\begin{tabular}{|c|c|c|c|c|c|c|c|c|c|}
\hline & \multicolumn{9}{|c|}{ Period } \\
\hline Agent & \multicolumn{3}{|c|}{1} & \multicolumn{3}{|c|}{2} & \multicolumn{3}{|c|}{3} \\
\hline 1 & \multirow{5}{*}{ 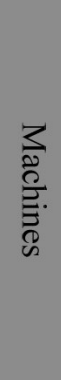 } & \multirow{5}{*}{ 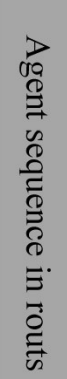 } & \multirow{5}{*}{ 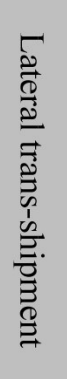 } & \multirow{5}{*}{ 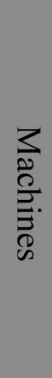 } & \multirow{5}{*}{ 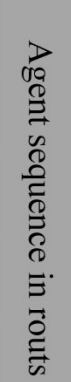 } & \multirow{5}{*}{ 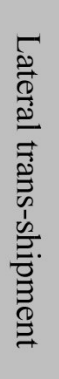 } & \multirow{5}{*}{$\begin{array}{l}3 \\
\text { है } \\
\text { : } \\
\text { : }\end{array}$} & \multirow{5}{*}{ 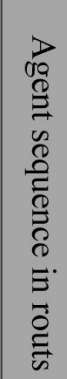 } & \multirow{5}{*}{ 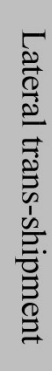 } \\
\hline 2 & & & & & & & & & \\
\hline 3 & & & & & & & & & \\
\hline 4 & & & & & & & & & \\
\hline 5 & & & & & & & & & \\
\hline
\end{tabular}

Figure 4. Proposed chromosome as a single line chromosome 


\subsubsection{Mutation}

Mutation is the agent of the GA not to be stuck in local optimization. Though it does not seem to be an important operator, lack of an eligible mutation probably leads the algorithm to being not effective. Normally, the mutation operator harshly and completely randomly changes the entrance chromosome as long as it remains feasible. Because of abstruseness of the proposed chromosome, there are several ways approaching randomized alteration of chromosome by which we are sure about the chromosome feasibility. We introduce two different mutation operators that make our methodology powerful enough to evade sticking in probable local optimum.

In explanation of applied mutation operator we use the mentioned 1th, 2th, and 3th column in crossover section. This paper methodology uses equally randomly the two distinct mutations. The following is their introductions:

- Choose between mutation 1 or 2 by equal chance

- Mutation 1

1. Produced as many as $3 * n P e r i o{ }^{*} m u$ randomized numbers. (mu: mutation rate)

2. Cheek for each produced number indicate to which column kind (1th: $0,3,6,9, \ldots$ 2 th: $1,4,7, \ldots$ th: $2,5,8, \ldots)$

3. For each specified column by produced random number generate a whole new column with respect to the kind of column and the other problem specifications.

- Mutation 2

1. Produced as many as $3{ }^{*} n$ Period $^{*} n A g e n t^{*} m u$ pair of randomized numbers. (mu: mutation rate)

2. Cheek for the first of each pair of produced number indicate to which column kind (1th: $0,3,6,9, \ldots 2$ th: $1,4,7, \ldots 3$ th: $2,5,8, \ldots$ )

3. If the first number indicate to 1 th column simply produce randomly a new number which is the representative of a machine to replace the indicated number in the chromosome

4. If the first number indicate to 2 th column simply produce randomly two number which is the representative of an agent and simply switch the two numbers is indicated by them

5. If the first number indicate to 3th column simply produce randomly a new number which is the representative of an agent to replace the indicated number in the chromosome 


\subsection{Selection and constant specifying}

In order to select chromosome to undergo mutation or crossover we simply omit a proportion of population by pNew rate and randomly by equal chances choose one or two chromosome with respective to the operation, crossover or mutation. Furthermore, because the essence of this paper presented chromosome, crossover or mutation, for every iteration some new chromosome, by the rate of pNew, is initiated. In the process of solving we specified 30, 30, $0.7,0.1,0.3$, and 0.1 respectively for number of population, number of iterations, crossover percentage, mutation percentage, mutation rate, and new chromosome percentage ( $\mathrm{pNew}$ ).

\subsection{Fitness function calculation}

As we mentioned earlier, because of the mentioned abstruseness of multi item IRP problem, the process of calculating fitness function in this paper is amalgamated with running PSO process, in another word in every fitness function calculation the PSO process is once run. For the sake of keeping our proposed methodology applicable in matter of time consumption we just took the advantage of PSO initialization process. GA chromosome, as it mentioned in decoding function just specify two of our decision variables, that is $X_{i j k t}$ and $Y_{i j t}$ respectively machine kind 1 and lateral trans-shipment routing. Specifying other decision variables, that is, $I_{i s t}, B_{i s t}, q_{i s k t}$ and $T_{i j s t}$ is the quite massive burden of PSO procedure. Proposed PSO procedure randomly and with respect to the problem constraints such as Machine and agents capacity specify those unspecified decision variables. Finally, when all of the decision variables are specified fitness value is calculated, using Equation (1) objective.

\section{Proposed Methodology Output and Financial Analysis}

In order to show the applicability and usefulness of the proposed model and solution method, we provide some illustrative examples. To generate the parameters we used uniform distributions. Table 2 shows the range used for generating each parameter. Moreover, we assumed that lateral trans-shipment costs are derived from following equation with $a=0.1$. Also, $Q 1$ and $Q 2$ are assumed, respectively, to be 6,000 and 4,000.

$$
p_{i j}=(1+\alpha) c_{i j}
$$




\begin{tabular}{|c|c|}
\hline Parameter & Range \\
\hline$t$ & {$[1,20]$} \\
\hline$N$ & {$[1,20]$} \\
\hline$k$ & {$[1,10]$} \\
\hline$s$ & {$[1,6]$} \\
\hline$P_{s}$ & {$[1,3]$} \\
\hline$d_{i s t}$ & {$[600,4000 / \mathrm{s}]$} \\
\hline$c_{i j}$ & {$[50,200]$} \\
\hline$V_{i}$ & {$[3000,5000]$} \\
\hline$h_{i}$ & {$[0.06,0.1]$} \\
\hline$\pi_{i}$ & {$[0.03,0.1]$} \\
\hline$t^{\prime}$ & {$[0.20,0.25]$} \\
\hline$f_{t}$ & {$[0.10,0.15]$} \\
\hline
\end{tabular}

Table 2. Range for random generation of parameters

Table 3 shows the influence of problem dimensions on proposed methodology demeanor and Letters ' $S$ ', 'M', and ' $L$ ' in the second and third column of this table respectively stand for Small, Medium and Large. This table's aim is to illustrate the influence of Number of Period and Number of Agents on the time of solving. As one can distinguish number of period is a more impacting variable than number of agent on the time of solving. Table 4 shows the comparison between GAMS and proposed methodology results. Note that, due to this paper assumption of existing shortage all of the agents in each period may be given a very different number of products. It can differ from zero to the number of vehicle capacity or the number of agent's capacity. Therefore, the number of possible solutions even for small problems is infinite and it is logically wrong to consider that the proposed algorithm may result optimum solution. However it is tangibly noticeable that the algorithm finishes in reliable answers.

\begin{tabular}{|c|c|c|c|c|c|c|c|c|c|}
\hline & \multicolumn{2}{|c|}{ No. Agent } & \multicolumn{2}{|c|}{ No. Period } & No. Items & No. Vehicle & No. Iteration & $\begin{array}{c}\text { Time } \\
\text { consumption }\end{array}$ & $\begin{array}{c}\text { Proposed } \\
\text { methodology }\end{array}$ \\
\hline 1 & S & 3 & S & 3 & 4 & 4 & 30 & $9.7 \mathrm{~s}$ & 28388.6 \\
\hline 2 & S & 5 & M & 10 & 5 & 4 & 30 & $69 \mathrm{~s}$ & 29494.6 \\
\hline 3 & S & 2 & L & 17 & 5 & 5 & 30 & $122.6 \mathrm{~s}$ & 27355.1 \\
\hline 4 & M & 7 & S & 5 & 4 & 6 & 30 & $41.9 \mathrm{~s}$ & 21461.8 \\
\hline 5 & M & 8 & M & 9 & 5 & 3 & 30 & $98.9 \mathrm{~s}$ & 20793.7 \\
\hline 6 & M & 11 & L & 20 & 5 & 5 & 30 & $324.4 \mathrm{~s}$ & 16234.3 \\
\hline 7 & L & 17 & S & 6 & 5 & 4 & 30 & $169.5 \mathrm{~s}$ & 11714.3 \\
\hline 8 & L & 19 & M & 8 & 5 & 7 & 30 & $251.7 \mathrm{~s}$ & 12842.2 \\
\hline 9 & L & 20 & L & 19 & 4 & 8 & 30 & $528.5 \mathrm{~s}$ & 12799.6 \\
\hline
\end{tabular}

Table 3. Influence of problem dimensions on proposed methodology demeanor 


\begin{tabular}{|c|c|c|c|c|c|c|c|c|}
\hline & $\begin{array}{c}\text { No. } \\
\text { Agent }\end{array}$ & No. Period & No. Items & $\begin{array}{c}\text { No. } \\
\text { Vehicle }\end{array}$ & GAMS Result & $\begin{array}{c}\text { Average } \\
\text { Proposed } \\
\text { methodology }\end{array}$ & $\begin{array}{c}\text { Best Proposed } \\
\text { methodology }\end{array}$ \\
\hline 1 & 5 & 1 & 1 & 1 & 9239.2 & 8844.2 & 8953.4 & 3.09 \\
\hline 2 & 9 & 1 & 1 & 8 & 13504.5 & 13015.3 & 13342.7 & 1.20 \\
\hline 3 & 3 & 5 & 1 & 3 & 29822.4 & 28678.6 & 29249.1 & 1.92 \\
\hline 4 & 12 & 10 & 3 & 7 & 60218.0 & 59542.9 & 59813.2 & 0.67 \\
\hline 5 & 17 & 5 & 2 & 4 & 32448.3 & 31449.5 & 31527.4 & 2.84 \\
\hline 6 & 7 & 5 & 2 & 5 & 34227.2 & 33075.8 & 34120.3 & 0.31 \\
\hline 7 & 8 & 2 & 2 & 3 & 11110.0 & 9743.4 & 10627.9 & 4.34 \\
\hline 8 & 9 & 4 & 2 & 5 & 31815.6 & 30561.1 & 30965.3 & 2.67 \\
\hline 9 & 5 & 4 & 2 & 1 & 19001.9 & 18583.0 & 18586.1 & 2.19 \\
\hline 10 & 9 & 1 & 1 & 4 & 6416.8 & 5711.3 & 6281.7 & 2.11 \\
\hline 11 & 3 & 4 & 2 & 1 & 22963.1 & 20475.2 & 21517.1 & 6.30 \\
\hline 12 & 11 & 5 & 3 & 1 & 33331.7 & 31184.4 & 32943.0 & 1.17 \\
\hline 13 & 10 & 1 & 2 & 9 & 6369.3 & 5424.6 & 5733.5 & 9.98 \\
\hline 14 & 9 & 3 & 3 & 1 & 28901.6 & 27851.3 & 28382.9 & 1.79 \\
\hline 15 & 19 & 5 & 4 & 13 & 47511.6 & 46146.0 & 46254.3 & 2.65 \\
\hline & & & & & & & Average gap: \\
\hline
\end{tabular}

Table 4. Comparison between GAMS and Proposed Methodology

So far in this paper, a new methodology is proposed for those companies who produce several products and have a set of agents in a particular city to decide optimally about the routing and inventory matters in all of their agents. With considering the revenue of each product, inventory, shortage, transportation costs and also inflection and interest rates, the proposed methodology will specifically return the net present income of the company business. As it is mentioned before, the main concern of this paper is to help an organization to decide economically whether establish a new agent. To this end, decision maker(s) should solve the problem in following three phases:

- Solving proposed IRP model and calculating net present income of the company in the desired horizon without considering new agent in the model.

- Solving proposed IRP model and calculating net present income of the company in the desired horizon with considering new agent in the model.

- Comparing charges of establishing the new agent and difference between net present income of the company with considering new agent and without it. If the difference between net present incomes in two cases is greater than charges of establishing the new agent, establishing new company has economic justification.

\section{Discussion and Conclusion}

This study investigates a multi-item, multi-period IRP, where each Agent's demand can be fulfilled directly by the firm itself and indirectly by other agents through lateral trans-shipments. The main concern of this research is to propose a practical IRP model considering simultaneously 
lateral trans-shipment and financial decisions in order to help an organization to decide economically whether establish a new agent. Consequently, a MILP model is proposed with the aim of maximizing net present income of the firm regarding purchasing income and its various costs. To solve the proposed model a new hybrid GA-PSO meta-heuristic algorithm is introduced. To demonstrate the usefulness of the proposed hybrid algorithm some randomly generated test problem was provided, the comparison between hybrid algorithm results and GAMS solutions indicated that the average gap is 2.88 percent and the hybrid gives totally reliable and good answers. The most notable upshot can be drawn out of the depicted result is that the proposed hybrid can be applied to real and big problems since GAMS cannot deal with such problems. Finally, a decision procedure with three phases is proposed to help an organization to find whether establishing a new agent has economic justification or not.

Although the hybrid model can produce entitled solutions, it should be noted that a metaheuristic performance strongly relies on the parameters of it. The setting of parameters is one of the most popular subjects of current research in meta-heuristics. Future research can be base on developing a methodology to tune the proposed hybrid algorithm's parameters.

\section{References}

Abdelmaguid, T.F., Dessouky, M.M., \& Ordóñez, F. (2009). Heuristic approaches for the inventoryrouting problem with backlogging. Computers \& Industrial Engineering, 56(4), 1519-1534. http://dx.doi.org/10.1016/j.cie.2008.09.032

Andersson, H., Hoff, A., Christiansen, M., Hasle, G., \& Løkketangen, A. (2010). Industrial aspects and literature survey: Combined inventory management and routing. Computers \& Operations Research, 37(9), 1515-1536. http://dx.doi.org/10.1016/j.cor.2009.11.009

Bard, J.F., \& Nananukul, N. (2010). A branch-and-price algorithm for an integrated production and inventory routing problem. Computers \& Operations Research, 37(12), 2202-2217. http://dx.doi.org/10.1016/j.cor.2010.03.010

Bertazzi, L., Bosco, A., Guerriero, F., \& Laganà, D. (2011). A stochastic inventory routing problem with stock-out. Transportation Research Part C: Emerging Technologies.

Campbell, A.M., \& Savelsbergh, M.W.P. (2004). A Decomposition Approach for the InventoryRouting Problem. Transportation Science, 38, 488-502. http://dx.doi.org/10.1287/trsc.1030.0054

Chen, Y.M., \& Lin, C.-T. (2009). A coordinated approach to hedge the risks in stochastic inventory-routing problem. Computers \& Industrial Engineering, 56(3), 1095-1112. http://dx.doi.org/10.1016/j.cie.2008.09.044

Coelho, L.C., Cordeau, J.-F., \& Laporte, G. (2012). The inventory-routing problem with transshipment. Computers \& Operations Research, 39(11), 2537-2548. 
Eberhart, R.C., \& Shi, Y. (2001). Particle swarm optimization: Developments, applications and resources.

Fredrik, O. (2009). Optimal policies for inventory systems with lateral transshipments. International Journal of Production Economics, 118(1), 175-184. http://dx.doi.org/10.1016/j.ijpe.2008.08.021

Fredrik, O. (2010). An inventory model with unidirectional lateral transshipments. European Journal of Operational Research, 200(3), 725-732. http://dx.doi.org/10.1016/j.ejor.2009.01.024

Kennedy, J., \& Eberhart, R. (1995). Particle swarm optimization.

Lenstra, J.K., \& Kan, A.H.G.R. (1981). Complexity of vehicle routing and scheduling problems. Networks, 11(2), 221-227. http://dx.doi.org/10.1002/net.3230110211

Liu, S.-C., \& Chen, J.-R. (2011). A heuristic method for the inventory routing and pricing problem in a supply chain. Expert Systems with Applications, 38(3), 1447-1456. http://dx.doi.org/10.1016/j.eswa.2010.07.051

Moin, N.H., \& Salhi, S. (2007). Inventory Routing Problems: A Logistical Overview. The Journal of the Operational Research Society, 58(9), 1185-1194. http://dx.doi.org/10.1057/palgrave.jors.2602264

Paterson, C., Kiesmuller, G., Teunter, R., \& Glazebrook, K. (2011). Inventory models with lateral transshipments: A review. European Journal of Operational Research, 210(2), 125-136. http://dx.doi.org/10.1016/j.ejor.2010.05.048

Prins, C. (2004). A simple and effective evolutionary algorithm for the vehicle routing problem. Computers \& Operations Research, 31(12), 1985-2002. http://dx.doi.org/10.1016/S03050548(03)00158-8

Shen, Q., Chu, F., \& Chen, H. (2011). A Lagrangian relaxation approach for a multi-mode inventory routing problem with transshipment in crude oil transportation. Computers \& Chemical Engineering, 35(10), 2113-2123. http://dx.doi.org/10.1016/j.compchemeng.2011.01.005

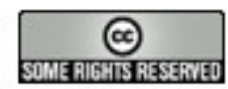

Article's contents are provided on a Attribution-Non Commercial 3.0 Creative commons license. Readers are allowed to copy, distribute and communicate article's contents, provided the author's and Journal of Industrial Engineering and Management's names are included. It must not be used for commercial purposes. To see the complete license contents, please visit http://creativecommons.org/licenses/by-nc/3.0/. 